\title{
ON THE ALMOST SURE CONVERGENCE OF WEIGHTED SUMS OF RANDOM ELEMENTS IN D[0,1]
}

\author{
R.L. TAYLOR and C.A. CALHOUN \\ Department of Mathematics and Statistics \\ University of South Carolina \\ Columbia, S. C. 29208
}

(Received June 26, 1980 and in revised form February 17, 1981)

ABSTRACT. Let $\left\{w_{n}\right\}$ be a sequence of positive constants and $w_{n}=w_{1}+\ldots+w_{n}$ where $w_{n} \rightarrow \infty$ and $w_{n} / W_{n} \rightarrow \infty$. Let $\left\{W_{n}\right\}$ be a sequence of independent random elements in $D[0,1]$. The almost sure convergence of $w_{n}^{-1} \sum_{k=1}^{n} w_{k} X_{k}$ is established under certain integral conditions and growth conditions on the weights $\left\{w_{n}\right\}$. The results are shown to be substantially stronger than the weighted sums convergence results of Taylor and Daffer (1980) and the strong laws of large numbers of Ranga Rao (1963) and Daffer and Taylor (1979).

KEY WORDS AND PHRASES. Weighted Sums, Random Elements in D 0,1$]$, Strong Laws of Large Numbers, Integral Conditions, and Almost Sure Convergence.

1980 MATHEMATICS SUBJECT CLASSIFICATION CODES. Primary 60B12; secondary 60F15.

1. INTRODUCTION.

The convergence of weighted sums of random elements in $\mathrm{D}[0,1]$ was obtained by Taylor and Daffer (1980) using a number of conditions such as convex tightness, moment conditions, and others. In this paper both the moment conditions and the tightness conditions are substantially relaxed in obtaining the almost sure convergence of weighted sums of random elements in $D[0,1]$. In addition, a brief discussion of the necessary integral conditions will be included which will delineate these results and previous work. 
Let $D[0,1](=D)$ denote the space of real-valued functions on $[0,1]$ which are right continuous and possess left-hand limits for each $t \in[0,1]$. Let the linear space D be equipped with the topology generated by the Skorohod metric d and let \|\|$_{\infty}$ denote the uniform norm, $\|x\|_{\infty}=\sup _{t \in[0,1]}|x(t)|$ for $x \in$ D. Next, let $(\Omega, A, P)$ denote a probability space. A random element $X$ in $D$ is a function $\mathrm{X}: \Omega \rightarrow \mathrm{D}$ which is measurable with respect to the Borel sets of the Skorohod topology. Random elements in D are characterized by the property that $X(t)$ is a random variable for each $t \in[0,1]$. The expected value $E X \in D$ can be defined pointwise by $(E X)(t)=E(X(t))$ for each $t \in[0,1]$ when $E\|X\|_{\infty}<\infty$. Detailed geometric and probabilistic properties of the space D with the uniform norm and the Skorohod metric can be found in Billingsley (1968), pp. 109-153 and Taylor (1978), pp. 153-184.

\section{INTEGRAL CONDITIONS.}

In obtaining the strong law of large numbers for independent, identically distributed random elements in D, Ranga Rao (1963) used the following crucial lemma.

LEMMA 1. If $X$ is a random element in $D$ with $E\|X\|_{\infty}<\infty$, then for each $\varepsilon>0$ there exists a partition $0=t_{0}<t_{1}<\ldots<t_{m}=1$ of $[0,1]$ such that

$$
\max _{1 \leq i \leq m} \sup _{t-1 \leq t, s<t} E|X(t)-X(s)| \leq \varepsilon
$$

In extending inequality (2.1) to a sequence of random elements and in trying to obtain strong laws of large numbers for non-identically distributed random elements, the following formulation of integral properties were motivated. In each of the following properties and in Lemma 1 , the partition $\left\{t_{1}, \ldots, t_{m}\right\}$ depends on $\varepsilon$.

DEFINITION. A sequence of random elements $\left\{X_{n}\right\}$ in $D$ is said to satisfy (a) property (RT) if for each $\varepsilon>0$ there exists a partition $0=t_{0}<t_{1}<\ldots<t_{m}=1$ of $[0,1]$ such that 


$$
\sup _{n} \max _{0 \leq i \leq m-1} E\left[\left|x_{n}\left(t_{i+1}-0\right)-x_{n}\left(t_{i}\right)\right|\right] \leq \varepsilon
$$

(b) property $(\mathrm{mT})$ if for each $\varepsilon>0$ there exists a partition $0=t_{0}<t_{1}<\ldots<t_{m}=1$ of $[0,1]$ such that

$$
\sup _{n} \max _{0 \leq 1 \leq m-1} E\left[\sup _{t_{i} \leq t<t}\left|x_{n}(t)-x_{n}\left(t_{i}\right)\right|\right] \leq \varepsilon .
$$

(c) property ( $T$ ) if for each $\varepsilon>0$ there exists a (Skorohod) compact set $\mathrm{K}$ such that

$$
\sup _{\mathrm{n}} \mathrm{E}\left\|\mathrm{X}_{\mathrm{n}} \mathrm{I}_{\left[\mathrm{X}_{\mathrm{n}} \in \mathrm{K}\right]}\right\|_{\infty} \leq \varepsilon
$$

Clearly, property (mT) implies property (RT). Also, identically distributed random elements $\left\{\mathrm{X}_{n}\right\}$ with $E\left\|\mathrm{X}_{1}\right\|_{\infty}<\infty$ satisfy (RT) obviously and satisfy (mT) by Lemma 2 since $(T)$ follows from the tightness of identical distributions.

LEMMA 2. If $\left\{x_{n}\right\}$ satisfies property (RT) and property ( $\left.T\right)$, then $\left\{x_{n}\right\}$ satisfies property (mT).

PROOF. Let $\varepsilon>0$ be given, Choose $\mathrm{K}$ (Skorohod) compact such that

$$
\left.\sup _{n} E \| X_{n} I_{\left[X_{n}\right.} \in K\right] \|_{\infty} \leq \varepsilon / 6
$$

Since $\mathrm{K}$ is compact, there exists $\delta>0$ such that

$$
|x(t)-x(s)| \leq|x(u-0)-x(s)|+\varepsilon / 3
$$

for each $\mathrm{x} \in \mathrm{K}$ whenever $0 \leq s \leq t<u<s+\delta$.

By (RT) choose a partition $\left\{t_{1}, \ldots, t_{m}\right\}$ of $[0,1]$ such that

$$
\sup _{n} \max _{i} E\left[\left|x_{n}\left(t_{i+1}-0\right)-x_{n}\left(t_{i}\right)\right|\right] \leq \varepsilon / 3 \text {. }
$$

Without loss of generality it can be assumed that $t_{i+1}-t_{i}<\delta$ for all $i=0,1, \ldots, m-1$. Thus, from (2.6) and (2.5)

$$
\sup _{n} \max _{i} E\left[\sup _{t_{i} \leq t<t}\left|x_{n}(t)-x_{n}\left(t_{i}\right)\right|\right]
$$




$$
\begin{aligned}
& \leq \sup _{n} \max _{i} E\left[\sup _{t_{i} \leq t<t_{i+1}}\left|x_{n}(t)-x_{n}\left(t_{i}\right)\right| I_{\left[x_{n} \in K\right]}\right] \\
& \left.+\sup _{n} \max _{i} E\left[\sup _{t_{i} \leq t<t}\left|x_{n}(t)-x_{n}\left(t_{i}\right)\right| I_{\left[x_{n}\right.} \notin k\right]\right] \\
& \leq \sup _{n} \max _{i} E\left[\left(\left|X_{n}\left(t_{i+1}-0\right)-X_{n}\left(t_{i}\right)\right|+\varepsilon / 3\right) I_{\left[X_{n} \in K\right.}\right] \\
& +\sup _{n} E\left[2\left\|x_{n}\right\|_{\infty} I_{\left[x_{n} \notin K\right]}\right] \\
& \leq \sup _{n} \max _{i} E\left[\left|X_{n}\left(t_{i+1}-0\right)-X_{n}\left(t_{i}\right)\right| I_{\left[X_{n} \in K\right.}\right]+\varepsilon / 3+\varepsilon / 3 \\
& \leq \varepsilon / 3+\varepsilon / 3+\varepsilon / 3=\varepsilon
\end{aligned}
$$

by $(2.7)$.

Property $(T)$ has been used in several laws of large numbers for random elements in Banach spaces to eliminate the assumption of identical distributions. In obtaining laws of large numbers for D, Daffer and Taylor (1980) required convex tightness or that the (Skorohod) compact set in $(2.4)$ also be convex. In particular, the previous convex tightness condition can be partially formulated by the integral condition

$$
\sup _{n} E\left[\max _{i} \sup _{t_{i} \leq t<t_{i+1}}\left|x_{n}(t)-x_{n}\left(t_{i}\right)\right|\right] \leq \varepsilon .
$$

Property $(\mathrm{mT})$ is clearly much more general than $(2.8)$, and convergence results in the next section using property $(\mathrm{mT})$ are a marked improvement over the results of Taylor and Daffer (1980) and include the results for identical distributions.

\section{ALMOST SURE CONVERGENCE.}

Let $\left\{w_{k}\right\}$ denote a sequence of positive constants. Define $w_{n}=w_{1}+\ldots+w_{n}, s_{n}=\sum_{k=1}^{n} w_{k} x_{k}$, and $N(x)=\operatorname{card}\left\{n: w_{n}^{-1} w_{n} \leq x\right\}$. Random variables $\left\{x_{n}\right\}$ are said to have uniformly bounded tail probabilities if there exists a nonnegative real-valued random variable $\mathrm{X}$ such that $P\left[\left|X_{n}\right|>t\right] \leq P[X>t]$ for each $n$ and for all $t \in R$. This property is denoted by $\left\{x_{n}\right\} \propto x$. The following real-valued result can be obtained from the geometric 
Banach space results of Howell, Taylor and Woyczynski (to appear) and will be used in establishing the almost sure convergence of weighted sums of random elements in D.

THEOREM 1. Let $\left\{\mathrm{x}_{\mathrm{n}}\right\}$ be independent (real-valued) random variables such that $\left\{x_{n}\right\} \propto x, E N(X)<\infty$, and for some $1 \leq p \leq 2$

$$
\int_{0}^{\infty} t^{P-1} P[X>t] \int_{t}^{\infty} y^{-(P+1)} N(y) d y d t<\infty .
$$

If $w_{n} \rightarrow \infty$, then

$$
w_{n}^{-1} s_{n}-c_{n} \rightarrow 0 . \quad \text { a.s. }
$$

where

$$
c_{n}=w_{n}^{-1} \sum_{k=1}^{n} w_{k} E\left(X_{k} I_{\left[\left|x_{k}\right| \leq w_{k}^{-1} w_{k}\right]}\right)
$$

REMARK. If $w_{n}^{-1} w_{n} \rightarrow \infty, E X<\infty$, and $\operatorname{EX}_{1}=\operatorname{EX}_{n}$ for all $n$, then

$$
\mathrm{W}_{\mathrm{n}}^{-1} \mathrm{~S}_{\mathrm{n}}-\mathrm{EX}_{1} \rightarrow 0 \quad \text { a.s. }
$$

since

$$
\begin{aligned}
& \quad \operatorname{EX}_{n}-\operatorname{Ex}_{n} I_{\left[\left|X_{n}\right| \leq w_{n}^{-1} W_{n}\right]} \mid=\int_{w_{n}^{-1} W_{n}}^{\infty} t d P\left[\left|X_{n}\right| \leq t\right] d t \\
& =-\int_{w_{n}^{-1} W_{n}}^{\infty} t d P\left[\left|X_{n}\right|>t\right] \leq w_{n}^{-1} W_{n} P\left[X>w_{n}^{-1} W_{n}\right]+\int_{w_{n}}^{\infty} W_{n} P[X>t] d t \\
& \rightarrow 0 \text { as } n \rightarrow \infty
\end{aligned}
$$

THEOREM 2. Let $\left\{x_{n}\right\}$ be independent random elements in $D$ such that (i) property $(\mathrm{mT})$ is satisfied, (ii) $\left\{\left\|\mathrm{x}_{\mathrm{n}}\right\|\right\} \alpha \mathrm{X}$ and $\mathrm{EN}(\mathrm{X})<\infty$, (iii) $\mathrm{EX}_{\mathrm{n}}=\mathrm{EX}_{1}$ for all $\mathrm{n}$, and (iv) for some $1 \leq \mathrm{p} \leq 2$ Condition (3.1) holds. If $w_{n} \rightarrow \infty$ and $w_{n}^{-1} w_{n} \rightarrow \infty$, then

$$
\left\|W_{n}^{-1} s_{n}-E x_{1}\right\|_{\infty} \rightarrow 0 \quad \text { a.s. }
$$

PROOF. By property $(\mathrm{mT})$ there exists a partition $0=t_{0}<t_{1}<\ldots<t_{m}=1$ such that 


$$
\max _{0 \leq i \leq m-1} E\left[\sup _{t_{i} \leq t<t_{i+1}}\left|x_{k}(t)-X_{k}\left(t_{i}\right)\right|\right] \leq \varepsilon \text { for each } k \text {. }
$$

For each $\mathrm{n}$

$$
\begin{aligned}
& \left\|w_{n}^{-1} \sum_{k=1}^{n} w_{k} X_{k}-E x_{1}\right\|_{\infty} \\
& \leq \max _{0 \leq i \leq m-1} w_{n}^{-1} \sum_{k=1}^{n} \dot{w}_{k} \sup _{t_{i} \leq t<t_{i+1}}\left|x_{k}(t)-x_{k}\left(t_{i}\right)\right| \\
& \quad+\max _{0 \leq i \leq m}\left|w_{n}^{-1} \sum_{k=1}^{n} w_{k} x_{k}\left(t_{i}\right)-\operatorname{Ex}_{1}\left(t_{i}\right)\right| \\
& \quad+\max _{0 \leq i \leq m-1} t_{i} \leq t<t+1+\sup _{i+1}\left|\operatorname{Ex}_{1}(t)-\operatorname{Ex}_{1}\left(t_{i}\right)\right|
\end{aligned}
$$

Similar to the proofs in Taylor and Daffer (1980), the last term in (3.3) is less than $\varepsilon$ by property (mT). Also, noting that for each $i\left\{x_{k}\left(t_{i}\right)\right\} \alpha x$, the second term in (3.3) converges to 0 a.s. by Theorem 1. For the first term of (3.3), define

$$
Y_{k}^{(i)}=\sup _{t_{i} \leq t \leq t_{i+1}}\left|X_{k}(t)-x_{k}\left(t_{i}\right)\right| \text { and } z_{k}^{(i)}=Y_{k}^{(i)}-E Y_{k}^{(i)} \text {. }
$$

For each $i\left\{z_{k}^{(i)}\right\}$ are independent random variables with zero means and $\left\{z_{k}^{(i)}\right\} \propto 2 x$. Thus

$$
\mathrm{w}_{\mathrm{n}}^{-1} \sum_{\mathrm{k}=1}^{\mathrm{n}} \mathrm{w}_{\mathrm{k}} \mathrm{z}_{\mathrm{k}}^{(1)}+0 \quad \text { a.s. }
$$

by Theorem 1 , for each $i=0,1, \ldots, m-1$. Also, by Theorem 1

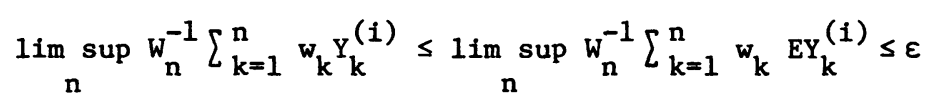

a.s. for each $i$ by property (mT). Hence,

$$
\underset{n}{\lim \sup }\left\|W_{n}^{-1} \sum_{k=1}^{n} w_{k} X_{k}-E X\right\|_{\infty} \leq \varepsilon \quad \text { a.s. }
$$

and the proof is completed by taking a sequence of $\varepsilon^{\prime}$ s going to zero and by excluding a countable union of null sets. 
To obtain the traditional strong law of large numbers, let $w_{k}=1$ for all $k$. Then, $N(y)=[y]$ (the greatest integer function), and condition (3.1) becomes approximately

$$
\int_{0}^{\infty} t^{p-1} P[x>t] \int_{t}^{\infty} y^{-(p+1)} y d y d t=\int_{0}^{\infty} P[x>t] d t
$$

for each $p>1$. Thus, the following corollary is immediate.

COROLLARY 1. If $\left\{X_{n}\right\}$ is a sequence of independent random elements in $D$ such that (i) property (mT) is satisfied, (ii) $\left\{\mathrm{X}_{\mathrm{n}}\right\} \alpha \mathrm{X}$ and $\mathrm{EX}<\infty$, and (iii) $\operatorname{EX}_{n}=\operatorname{EX}_{1}$, then

$$
\left\|n^{-1} \sum_{k=1}^{n} x_{k}-E x_{1}\right\| \rightarrow 0 \text { a.s. }
$$

It is easy to show that $\left\{x_{n}-E X_{n}\right\}$ has property (mT) if $\left\{x_{n}\right\}$ has property (mT). Also, if for some $p>1, E\left\|x_{n}\right\|^{P} \leq M$ for all $n$, then

$$
P\left[\left\|x_{n}\right\|>t\right] \leq t^{-P} M=\int_{t}^{\infty} s^{-(p+1)} p M d s
$$

will yield a random variable $X$ such that $\left\{x_{n}\right\} \propto x$ and

$$
E X=\int_{M}^{\infty} s^{-(p+1)} s p M d s=p M \int_{M^{-p}}^{\infty} s^{-p} d s<\infty .
$$

COROLLARY 2. If $\left\{X_{n}\right\}$ is a sequence of independent random elements in $D$ such that (i) property (mT) holds and (ii) for some $p>1$, sup $E\left\|x_{n}\right\|^{p}<\infty$, then

$$
\left\|n^{-1} \sum_{k=1}^{n} x_{k}-E x_{1}\right\|_{\infty} \rightarrow 0 \text { a.s. }
$$

Corollary 2 represents a major improvement over Theorem 1 of Daffer and Taylor (1979) in that not only is convergence in the Skorohod topology replaced by convergence in the uniform norm but the restrictive condition of convex tightness is replaced by property (mT). Finally, since identically distributed random elements with a first absolute moment satisfy property (mT), the following more general form of the strong law of large numbers is available.

THEOREM 3. If $\left\{x_{n}\right\}$ are identically distributed random elements in $D$ such 
that (i) $\mathrm{EX}_{1}$ exists and $\mathrm{EN}\left(\left\|\mathrm{X}_{1}\right\|\right)<\infty$ and (ii) condition (3.1) holds for $\left\|x_{1}\right\|=x$, then

$$
\left\|\mathrm{w}_{\mathrm{n}}^{-1} \sum_{k=1}^{\mathrm{n}} \mathrm{w}_{k} \mathrm{x}_{\mathrm{k}}-\mathrm{Ex}_{1}\right\| \rightarrow 0 \quad \text { a.s. }
$$

ACKNOWLEDGEMENTS. The authors are grateful to Peter $\mathrm{Z}$. Daffer for his discussions and help in the development of the integral conditions and their relationships, and to the referee for helpful comments on organization of the paper. The research was supported in part by the Air Force Office of Scientific Research under Contract number F49620-79-C-0140 and by the Research and Productive Scholarship Fund of the University of South Carolina.

\section{REFERENCES}

1. Billingsley, P. Convergence of Probability Measures, Wiley, New York, (1968).

2. Daffer, P. Z. and Taylor, R. L. Laws of large numbers for $D[0,1]$, Annals of Probability 7 (1979), 85-95.

3. Howe11, J., Taylor, R. L., and Woyczynski, W. A. Stability of linear forms in independent random variables in Banach spaces, (to appear) Proc. Prob. in Banach Spaces, III.

4. Rao, R. R. The law of large numbers for $D[0,1]$-valued random variables, Theory of Prob. and App1. 8 (1963), 70-74.

5. Taylor, R. L. Stochastic Convergence of Weighted Sums of Random Elements in Linear Spaces. Lecture Notes in Mathematics, 672. Springer-Verlag, Berlin, 1978.

6. Taylor, R. L. and Daffer, P. Z. Convergence of weighted sums of random elements in $\mathrm{D}[0,1]$, J. of Mult. Anal. 10 (1980), 95-106. 


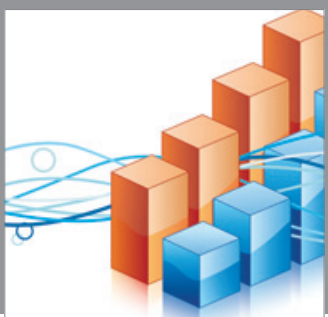

Advances in

Operations Research

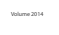

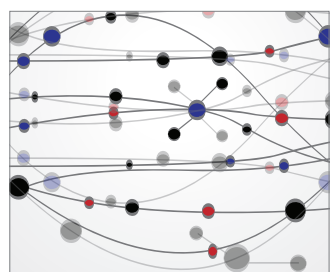

\section{The Scientific} World Journal
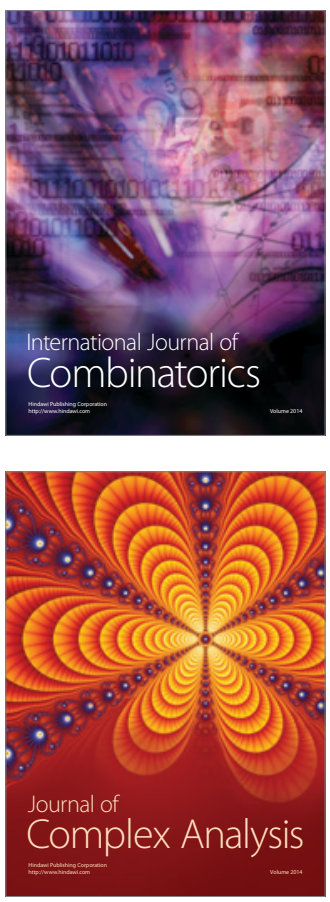

International Journal of

Mathematics and

Mathematical

Sciences
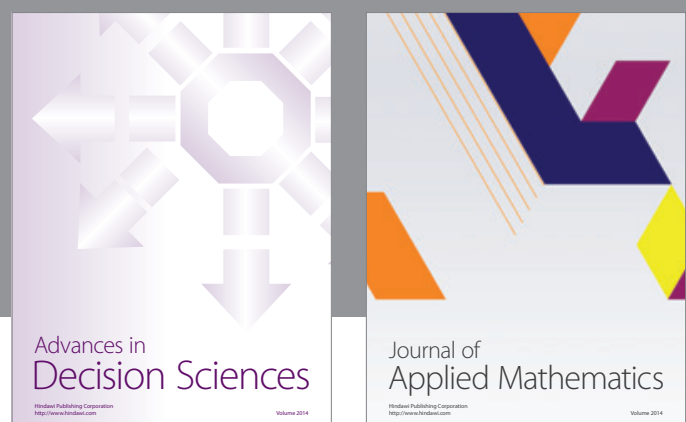

Journal of

Applied Mathematics
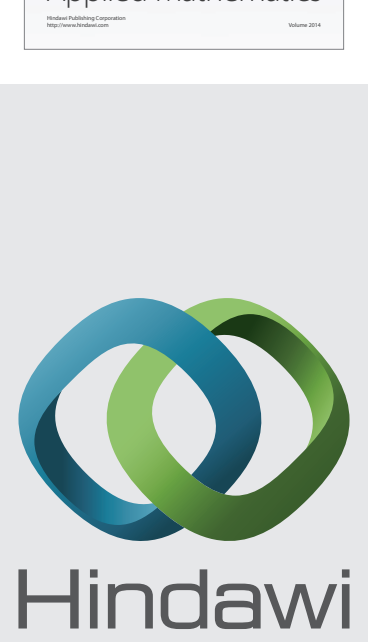

Submit your manuscripts at http://www.hindawi.com
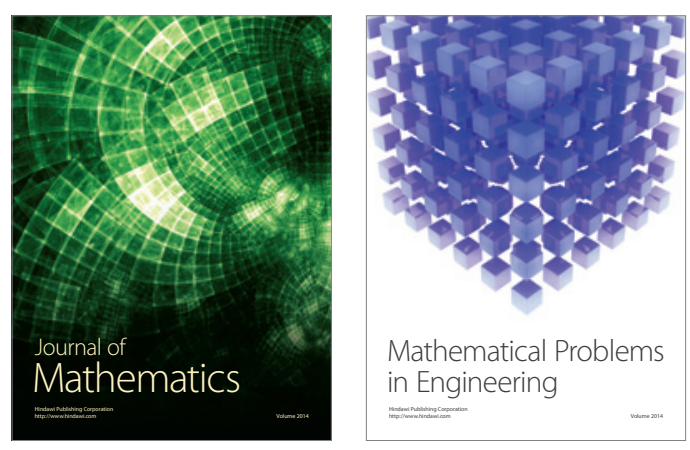

Mathematical Problems in Engineering
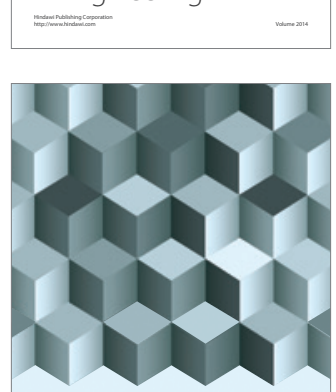

Journal of

Function Spaces
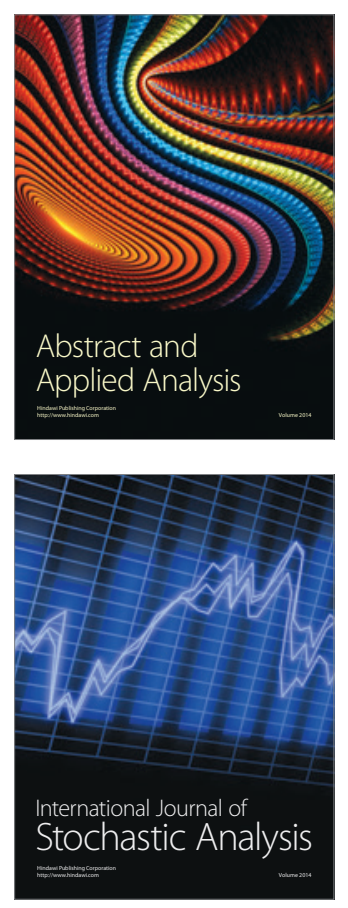

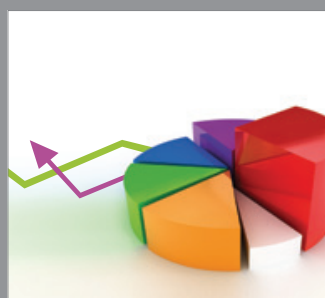

ournal of

Probability and Statistics

Promensencen
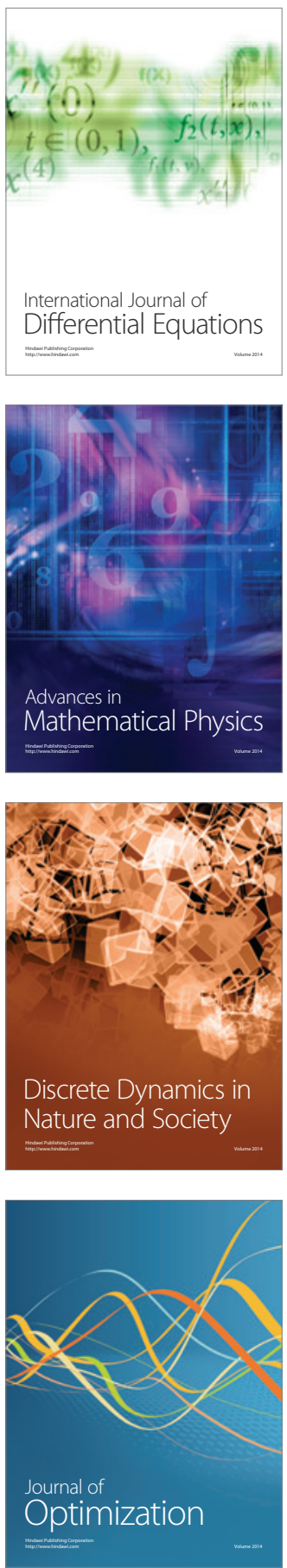